\title{
Comparison of the N-Terminal pro-Brain Natriuretic Peptide Levels, Neutrophil-to-Lymphocyte, Lymphocyte-to-Monocyte and Platelet-to-Lymphocyte Ratios Between the Patients with COVID-19 and Healthy Subjects; Are the Patients with COVID-19 Under the Risk of Cardiovascular Events?
}

\author{
Özgür Altınbaş ${ }^{1}$, İbrahim Halil Ertaş ${ }^{2}$, Ayşe Özlem Mete ${ }^{1}$, Şeniz Demiryürek ${ }^{1}$, Erhan \\ Hafiz $^{1}$, Ahmet Saracaloğlu ${ }^{1}$, and Abdullah Tuncay Demiryürek ${ }^{1}$ \\ ${ }^{1}$ Gaziantep University \\ ${ }^{2}$ Dr Ersin Arslan Training and Research Hospital
}

April 4, 2021

\begin{abstract}
Backround: The ongoing pandemic of Coronavirus Disease 2019 (COVID-19) infection has declared as a global emergency. Although the most people had mild illness due to the infection, some patients were seriously affected associated with complications especially including respiratory and cardiovascular systems. In despite of the respiratory system involvement is in the foreground, cardiovascular complications result in worse clinical outcomes. The purpose of this study is to compare the levels of the N-terminal pro-brain natriuretic peptide, neutrophil-to-lymphocyte, lymphocyte-to-monocyte and platelet-to-lymphocyte ratios -which are accepted as a marker of negative outcomes in cardiovascular diseases- of the patients with COVID-19 and healthy subjects and reveal the increased risk of cardiovascular disease in patients with COVID-19. Methods: This prospective study was designed in a single center and included 33 patients with COVID-19 (Group 1) and 33 healthy subjects consisted of health care professionals (Group 2). N-terminal pro-brain natriuretic peptide levels, neutrophil-to-lymphocyte, lymphocyte-to-monocyte and platelet-to-lymphocyte ratios were compared between the groups. Results: N-terminal pro-brain natriuretic peptide levels, neutrophil-to-lymphocyte and platelet-to-lymphocyte ratios were statistically significantly higher in the patients with COVID-19 $(\mathrm{p}<0,0001)$ and lymphocyte-to-monocyte ratio was statistically significantly lower in the same group ( $<00,0001)$. Conclusion: Cardiovascular manifestations result in worse outcomes in patients with COVID-19 although the disease primarily affects the respiratory system. Early detection of this clinical situation and taking precautions have vital importance in the progress of the disease. NT-proBNP levels, neutrophil-to-lymphocyte, lymphocyte-to-monocyte and platelet-to-lymphocyte ratios are inexpensive, easy, fast and reproducible parameters that can be used in determination of probabl cardiovascular events that may develop during COVID-19 and enables early optimal treatment strategies for patients.
\end{abstract}

Comparison of the N-Terminal pro-Brain Natriuretic Peptide Levels, Neutrophil-to-Lymphocyte, Lymphocyte-to-Monocyte and Platelet-to-Lymphocyte Ratios Between the Patients with COVID-19 and Healthy Subjects; Are the Patients with COVID-19 Under the Risk of Cardiovascular Events?

Abstract

Backround: The ongoing pandemic of Coronavirus Disease 2019 (COVID-19) infection has declared as a global emergency. Although the most people had mild illness due to the infection, some patients were seriously affected associated with complications especially including respiratory and cardiovascular systems. In despite of the respiratory system involvement is in the foreground, cardiovascular complications result 
in worse clinical outcomes. The purpose of this study is to compare the levels of the N-terminal probrain natriuretic peptide, neutrophil-to-lymphocyte, lymphocyte-to-monocyte and platelet-to-lymphocyte ratios -which are accepted as a marker of negative outcomes in cardiovascular diseases- of the patients with COVID-19 and healthy subjects and reveal the increased risk of cardiovascular disease in patients with COVID-19.

Methods: This prospective study was designed in a single center and included 33 patients with COVID-19 (Group 1) and 33 healthy subjects consisted of health care professionals (Group 2). N-terminal pro-brain natriuretic peptide levels, neutrophil-to-lymphocyte, lymphocyte-to-monocyte and platelet-to-lymphocyte ratios were compared between the groups.

Results: N-terminal pro-brain natriuretic peptide levels, neutrophil-to-lymphocyte and platelet-tolymphocyte ratios were statistically significantly higher in the patients with COVID-19 $(\mathrm{p}<0,0001)$ and lymphocyte-to-monocyte ratio was statistically significantly lower in the same group $(p<0,0001)$.

Conclusion: Cardiovascular manifestations result in worse outcomes in patients with COVID-19 although the disease primarily affects the respiratory system. Early detection of this clinical situation and taking precautions have vital importance in the progress of the disease. NT-proBNP levels, neutrophil-to-lymphocyte, lymphocyte-to-monocyte and platelet-to-lymphocyte ratios are inexpensive, easy, fast and reproducible parameters that can be used in determination of probabl cardiovascular events that may develop during COVID19 and enables early optimal treatment strategies for patients.

Keywords: Cardiovascular disease, COVID-19, NT-proBNP, lymphocyte-to-monocyte ratio, neutrophil-tolymphocyte ratio, platelet-to-lymphocyte ratio

What is already known about this topic?

. NT-proBNP levels, neutrophil-to-lymphocyte, lymphocyte-to-monocyte and platelet-to-lymphocyte ratios are related with inflammation which cause cardiac and vascular disorders in patients with COVID-19.

. NT-proBNP levels, neutrophil-to-lymphocyte, lymphocyte-to-monocyte and platelet-to-lymphocyte ratios are studied in literature but separately in patients with COVID-19.

What does this article add?

. This article is the unique in the literature in the subject of comparison of NT-proBNP levels, neutrophil-tolymphocyte, lymphocyte-to-monocyte and platelet-to-lymphocyte ratios between the patients with COVID19 and healthy population in one study.

\section{INTRODUCTION}

Coronavirus Disease 2019 (COVID-19) was accepted as a public health emergency situation and declared as a pandemic by the World Health Organization (WHO) at the beginning of the March 2019. ${ }^{1}$ A large number of countries and territories around the world were affected from the outbreak caused by severe acute respiratory syndrome coronavirus 2 (SARS-CoV-2). ${ }^{2}$ COVID-19 is the third disease caused by coronavirus within last two decades, after severe acute respiratory syndrome coronavirus (SARS-CoV) and Middle East respiratory syndrome coronavirus (MERS-CoV). ${ }^{3}$ Although lower case mortality rates of SARS-CoV-2, it has greater infectivity and has killed more people than those diseases. ${ }^{4}$

The clinical features of COVID-19 is mostly related with respiratory tract symptoms including cough, fever, pharyngodynia and complications related to pneumonia and acute respiratory distress syndrome. ${ }^{5}$ Most of the patients with COVID-19 had a good prognosis while critical patients might rapidly develop acute respiratory failure, acute respiratory distress syndrome, multipl organ failure and other mortal complications. ${ }^{6}$ These complications include; neurological deficits, gastrointestinal disorders, renal failure, myocarditis and acute coronary syndromes. ${ }^{3}$

Effects of COVID-19 on cardiovascular system is associated with COVID-19 induced multiple cytokines and chemokines resulting in plaque instability, vascular and myocardial inflammation. ${ }^{7}$ Cardiac involvement was 
found up to $20 \%$ of hospitalised patients with COVID-19 (1). Similarly, acute cardiac injury and arrhythmia were reported 7.2 and $16.7 \%$ respectively, in a single-center study consist of 138 COVID-19 patients. ${ }^{8}$ Huang et al. ${ }^{9}$ declared in their study that acute myocardial infarction was detected in $12 \%$ of patients with COVID19.

Several markers can be used in the assessment of cardiac injury among patients with COVID-19. One of these markers is N-terminal pro-brain natriuretic peptide (NT-proBNP) which is relased as a response to increased ventricular wall tension and shows reduced left ventricular systolic function and poor prognosis in cardiac invovement. ${ }^{10}$

Inflammation is one of the most important causes result in vascular damage. Beside various markers that indicate inflammation, neutrophil-to-lymphocyte (Neut/Lym), lymphocyte-to-monocyte (Lym/Mono) and platelet-to-lymphocyte (Plt/Lym) ratios are recently introduced biochemical markers for inflammationassociated diseases including vascular injury. ${ }^{11-13}$

Our study is uniqe in terms of comparing NT-proBNP levels, Neut/Lym, Lym/Mono and Plt/Lym ratios between the patients with COVID-19 and healthy subjects in the same study and we aimed to reveal the incresed risk of cardiovascular diseases among patients with COVID-19 by using these parameters.

\section{MATERIALS AND METHOD}

Between October 2020 and January 2021, 33 adult patients diagnosed with COVID-19 and 33 healthy subjects were included to this study. Diagnose of the disease was performed via RNA detection from nasopharyngeal secretions with real-time reverse transcriptase polymerase chain reactions (PCR) and chest computed tomographic (CT) scan using the diagnostic criteria defined in the World Health Organization (WHO) interim guidance.Healthy subjects were consisted entirely of healthcare professional volunteers without any chronical disease including cardiovascular disease, cerebrovascular disease, hypertension, diabetes mellitus, chronic obstructive pulmonary disease and any kind of neoplasia, working in the institute in which the study was designed. COVID-19 was excluded with the absence of viral RNA in PCR test results in those group. Written informed consent was taken from all participants and this single center prospective study was approved by the Clinical Ethics Committee of the institution (decision no: 2020/369) and complies with the declaration of Helsinki.

Data of the patients including demographic characteristics (age, gender), comorbidities, laboratory findings, radiographic images, electrocardiographic findings and treatments were collected from hospital records. Laboratory tests were consist of blood count, liver and renal function analyses, C-reactive protein (CRP), D-Dimer and NT-proBNP which were measured within 24 hours of admission. Complete blood cell counts and automated differential counts were determined via an automated hematology analyzer (Cell-Dyn 3700, Abbott, Germany) which provided total white blood cells count, platelet, neutrophil, lymphocyte, monocyte, eosinophil, and basophils counts/ml. The baseline Neut/Lym, Lym/Mono and Plt/Lym ratios were calculated by dividing the absolute counts of these parameters. An electrochemiluminescence-based immunoanalytical system, Elecsys 2010 (Roche Diagnostics Ltd, Manheim, Germany) was used to determine plasma levels of NT-proBNP.Patients with elevated NT-proBNP were hospitalized for close follow up to prevent and do early intervention for probabl cardiac disorders that may develop and all patients were performed transthoracic echocardiography. Favipiravir, hydroxychloroquine sulfate, levofloxacin and enoxaparine sodium were started after blood collection as an initial therapy to all patients according to the Turkish Health Ministery Guideline for COVID-19 Therapy.

Exclusion criteria of the patients with COVID-19 were any diagnosed cardiovascular and cerebrovascular diseases, hypertension, diabetes mellitus, chronic obstructive pulmonary disease, neoplasia,younger than 18 years of age, pregnant women and the primary end point of the study was the composite of death or the requirement of mechanical ventilation, intensive care unit or extracorporeal membrane oxygenation (ECMO).

Participants were divided into two groups; Group 1 was consisted of the patients with COVID-19 and the Group 2 was consisted of the healthy subjects. NT-proBNP levels, neutrophil-to-lymphocyte, lymphocyte- 
to-monocyte and platelet-to-lymphocyte ratios of the groups were compared.

Statistical Analyse

All values were shown as the mean $\pm \mathrm{SD}$ or percentage. The distribution of all variables was checked with the Kolmogorov-Smirnov test. Mann-Whitney U test was used for data with abnormal distribution.Otherwise, the differences between mean values of two groups were analyzed using the unpaired Student's t test. Gender of the two groups was analyzed using the chi-square test. The Pearson's test was used to assess the correlations, but Spearman correlation analysis was performed in correlation analysis of data with abnormal distribution. GraphPad Instat (version 3.05, GraphPad Software Inc., San Diego, CA, USA) statistical software was employed. The statistical significance was accepted at the level of 0.05 .

\section{RESULTS}

Thirty three patients with COVID-19 and 33 healthy subjects were included to the study. Participants were divided into two groups. The demographic features and laboratory findings were summarized in Table 1.There were no statistically significant differences in terms of demographic features including age and the gender between the groups.

There were also no marked differences in the subjects of leukocyte, neutrophil, monocyte, platelet counts and liver and kidney function tests. NT-pro BNP levels were markedly elevated in COVID-19 patients $(\mathrm{p}<0,0001)$ as shown in Figure 1. NT-pro BNP levels were 8,5 times higher in patients with COVID-19 than in normal population. D-Dimer $(\mathrm{p}=0,0135)$ and CRP $(\mathrm{p}<0,0001)$ levels and Neut/Lym $(\mathrm{p}<0,0001)$, Plt/Lym $(\mathrm{p}<0,0001)$ ratios are statistically significantly higher in Group 1. In contrast to these results, Lym/Mono ratio was found to be statistically significantly lower in Group $1(\mathrm{p}<0,001)$.

Correlations between ratios and biochemical parameters in patients with COVID-19 were also compared and results were given in Table 2 . We detected that there was positive correlation only between NT-proBNP and D-Dimer levels.

\section{DISCUSSION}

After the first cases describing pneumonia with unknown origin in Wuhan, China, SARS-CoV-2 rapidly became pandemic.Current epidemiologic studies suggest that about $80 \%$ of the patients with COVID-19 have mild symptoms while $5 \%$ become critically ill, requiring mechanical ventilation. ${ }^{14}$ Different type of responses are due to the degree of viral load, age of the patients, host immune response and prescence of comorbidities mainly hypertension, diabetes mellitus and thrombotic events. ${ }^{15}$ Although the most common symptoms including fever, dry cough, fatigue, headache, and shortness of breath areassociated with respiratory system and the complications were usually related to acute respiratory distress syndrome or pneumonia, cardiovascular involvement is also an additional clinical problem to deal with in COVID-19. ${ }^{16}$ Acute cardiac injury rates during the illness range between $7 \%$ to $28 \%$ in different studies which significantly increases the COVID-19 associated complications and mortality. ${ }^{17}$ Especially, presence of pre-existing cardiovascular disease and/or myocardial injury results in negative outcomes in patients with COVID-19. ${ }^{7}$ Increased affinity of the SARS-CoV-2 to angiotensin I converting enzyme 2 (ACE2) receptor which is expressed mainly in the lung and other tissues including vascular endothelial cells was shown in previous studies. ${ }^{18}$ Directly infection ability of SARS-CoV-2 to endothelial cells is one of the strange features of the virus and distinguishes it from the most of the infectious diseaes. ${ }^{19}$

Several mechanisms were suggested about the myocardial damage of SARS-CoV-2 including direct damage by the virus, systemic inflammatory responses, destabilized coronary plaque and aggravated hypoxia. ${ }^{9}$ Viral entry into the myocardium and arteries via ACE2 receptor induces ACE2 downregulation and reninangiotensin-aldosterone system dysfunction that leads to heart dysfunction and pneumonia progression. ${ }^{20}$ The activation and overproduction of inflammatory cytokines can also induce necrosis and apoptosis of cardiomyocyte. ${ }^{6}$ Imbalance of $\mathrm{T}$ helper 1 and $\mathrm{T}$ helper 2 responses triggered a cytokine storm in patients with COVID-19 which may cause myocardial injury. ${ }^{16}$ Vascular share stress of the coronary arteries can be increased by systemic inflammation which leads to plaque rupture and myocardial infarction. ${ }^{21}$ High oxygen 
demand due to tachycardia and fever and insufficient oxygen supply due to hypoxemia and respiratory failure are usually seen during the infection which could result in a type 2 myocardial infarction. ${ }^{22}$

Variety of cardiovascular involvement including heart failure, myocarditis, pericarditis, acute coronary syndrome and arrhythmias were described in previous studies. ${ }^{23}$ Although without a history of heart failure, new onset of heart failure was observed in hospitalized and intensive care required patients with COVID19 was observed in the rate of $25 \%$ and $30 \%$, respectively. ${ }^{24}$ Proinflammatory cytokines and recruitment of proinflammatory macrophages and granulocytes in blood stream lead to severe inflammatory storm and in combination with increased metabolic demand may cause cardiac depression and either new onset heart failure or acute decompensation of chronic heart failure. ${ }^{25}$ Viral infections have been shown as one of the most common infectious causes of myocarditis and pericarditis. In spite of widely reported myocarditis cases due to SARS-CoV-2, limited data have been published on cases with COVID-19 who developed pericarditis and pericardial effusion. ${ }^{26,27}$ Directly affected myocardium and pericardium via replicated and disseminated virus through the blood or the lymphatic system from the respiratory tract may be the probabl mechanism of these complications. ${ }^{5}$ In patients infected with SARS-CoV-2, increased interleukin- 6 and D-Dimer levels which indicates the augmented coagulation response, are linked with plaque instability and occurrence of acute coronary syndrome. ${ }^{28}$ According to the study designed by Bangalore et $\mathrm{al}^{29}, 9$ of the 18 patients with COVID-19 and had ST-segment elevation on electrocardiogram were performed coronary angiography. Three of them were found to be any obstructive disease which indicates hypoxic injury, coronary spasm, microthrombi and endothelial injury may also result in acute coronary syndrome without any occlusion in patients with COVID-19. According to a study designed in Wuhan it was revealed that acute coronary syndrome rates were significantly higher in 36 of the 138 patients with COVID-19 who were required intensive care. ${ }^{30}$ Cardiac arrhythmias and cardiac arrest are another common manifestation observed in COVID-19 patients but spesific types of arrhythmia were not described. High-grade atrioventricular tachycardia, atrial fibrillation, polymorphic ventricular tachycardia and pulseless electrical activity arrest are the common disorders mentioned in the literature. Hypoxemia and electrolyte disturbances are thought to be responsible from arrhythmogenicity. ${ }^{31,32}$

Several markers can be used in the detection of heart injury including hs-cTnI, NT-proBNP, CK-MB, myoglobin and lactate dehydrogenase. ${ }^{33}$ B-type natriuretic peptide (BNP) was first isolated from porcine brain, however it was soon found to originate mainly from the heart, representing a cardiac hormone. ${ }^{34} \mathrm{BNP}$ is synthesized as a prehormone (proBNP) consists of 108 aminoacids and on secretion this propeptide is split into its biologically active form BNP and the remaining NT-proBNP. Although plasma concentration and cardiac production of NT-proBNP is very low in the normal status, increased ventricular wall stretch, neurohormonal activation and hypoxia stimulate the secretion of this hormone. ${ }^{35}$

Increased levels of NT-proBNP and its association with worse outcomes in patients with COVID-19 were reported in several studies. In a study designed by Gao et $\mathrm{al}^{8}$, higher levels of NT-proBNP in patients with COVID-19 was found to be related with lower cumulative survival rate and its prognostic effect might be a specific index of reflecting the overall state of SARS-CoV-2 infection.Guo et al ${ }^{9}$ demonstrated a high positive correlation between troponin and NT-proBNP levels in COVID-19 patients in their study. Sorrentiono et $\mathrm{al}^{2}$ revealed that elevated NT-proBNP levels were associated with worse prognosis in COVID-19 patients, in their meta-analysis consisted of 2248 patients. Similarly, in an analysis including 416 hospitalized patients with COVID-19, NT-proBNP was significantly higher among patinets with myocardial injury than those without $^{36}$. Han et al ${ }^{4}$ indicated that NT-proBNP levels were significantly higher in severe and critical cases infected with SARS-CoV-2 than mild cases and can be used to predict the severity of the COVID-19. Zhou et $\mathrm{al}^{37}$ suggested that increased levels of NT-proBNP were associated with more severe symptoms and disease progression among patients with COVID-19. Mahajan et $a^{14}$ advocated that NT-proBNP has a critical role in estimating cardiac risk stratification and prognostication of patients with severe COVID-19. A metaanalysis by $\mathrm{Li}$ et $\mathrm{al}^{38}$, cardiac biomarkers included troponin, CK-MB and NT-proBNP were significantly higher in severe cases compared to milder cases.Abnormalities in NT-proBNP were found to be similar to the inflammatory markers in the subject of in-hospital mortality according to death risk analysis. ${ }^{39}$ Jin et $\mathrm{al}^{40}$ compared the NT-proBNP levels in patients with COVID-19 who developed cardiac injury and not, and 
demonstrated that patients with cardiac injury were more likely have elevation of NT-proBNP in comparison $66.7 \%$ to $10 \%$. Aladag and Atabey ${ }^{3}$ reported higher NT-proBNP levels in patients diagnosed with COVID-19 on the admission in correlation with our study. Our results demonstrated statistically significant increase in NT-proBNP levels in patients with COVID-19 when compared to the healthy population.

The role of the inflammation at every phase of atherosclerotic process was revealed in the literature.Immunoglobulin molecules, $\mathrm{T}$ lymphocytes, presence of antigen-antibody complex, and plasma cells that found in the structure of the atheroma plaque indicate the importance of the inflammation in the development and progression of atherosclerosis according to the histological studies. ${ }^{41}$

Different type of markers were defined as predictors of inflammation and cardiovascular risk in several studies such as; proinflammatory cytokines, adhesion molecules, oxidized low-density lipoproteins, acute phase reactants, white blood cells and erythrocyte sedimentation rate. ${ }^{42}$ In addition to these markers Neut/Lym, Lym/Mono and Plt/Lym ratios are novel biomarkers which are used as indicators of inflammation. ${ }^{11-13}$

Relationship between the white blood cells and its subtypes and presence and prognosis of atherosclerotic process was shown in several studies. Lymphocytes are one of its subtypes and responsible for cellular and humoral immunity. Lymphocyte apoptosis increases by the inflammation and lower lymphocyte counts are in correlation with worse outcomes in terms of vascular diseases. ${ }^{43}$

Neut/Lym ratio is a fast, easy and inexpensive method for assessment of inflammatory status and has been recently introduced biomarker for investigation of cardiovascular risk. Ratio of absolute neutrophil count to absolute lymphocyte count is the calculation method of this marker. ${ }^{44}$ The relation between atherosclerosis and Neut/Lym ratio was investigated by Balta et $\mathrm{al}^{45}$ and it was declared that Neut/Lym ratio can be affected by atherosclerotic risk factors such as hypertension, diabetes mellitus, hypercholesterolemia and metabolic syndrome; in addition it can also be used in the prediction of mortality in cardiovascular diseases. Similarly, correlation between Neut/Lym ratio and severity of coronary artery disease was shown in a study designed by Kaya et $\mathrm{al}^{46}$. Association between Neut/Lym ratio and COVID-19 studied in the literature and higher Neut/Lym ratio was found to be an independent risk factor of the in-hospital mortality for COVID-19 patients. ${ }^{47}$ In a study consisted of 125 patients with COVID-19 it was hypothesized that elevated Neut/Lym ratio would be a prognostic indicator of mortality in a COVID-19 patient population. ${ }^{48}$ In our study Neut/Lym values are also statistically significant higher in patients with COVID-19 than in control group.

Lym/Mono has been found to be a novel systemic inflammation marker which could reproducible and widely available in clinical praxis. Ratio of absolute lymphocyte count to absolute monocyte count is the calculation method of this marker. ${ }^{12}$ Decreased Lym/Mono ratio is found to be a risk factor for critical limb ischemia and other vascular peripheral arterial occlusive diseases in a study conducted by Gary et al. ${ }^{49}$ Demir et al ${ }^{50}$ suggested that lower Lym/Mono ratios are useful indicators for metabolic syndrome that includes atherosclerosis in vascular arterial structures. In a study designed by Lissoni et $\mathrm{al}^{51}, 71 \%$ of the patients with COVID-19 had lower Lym/Mono ratio when compared with controls, and they indicated that Lym/Mono is a simple and less expensive biomarker that can be used in clinical evolution of COVID-19 infection. Shivakumar et $\mathrm{al}^{52}$ compared the Lym/Mono ratios between the survivors and nonsurvivors of the patients with COVID-19 and Lym/Mono was lower in nonsurvivors group. Our study revealed statistically significantly lower values of Lym/Mono ratios in patients with COVID-19 than control group.

Plt/Lym ratio is one of the fast, broadly available and cheap marker that can be used in evaluation of inflammation and atherosclerosis. Ratio of absolute platelet count to absolute lymphocyte count is the calculation method of this marker. Its relation with severity and complexity of coronary artery disease in patients with acute coronary syndromes was studied and increased levels of Plt/Lym ratio was found in association with worse outcomes in those patients ${ }^{53}$. According to the study designed by Sari et al ${ }^{54}$ it was declared that patients with abnormal coronary angiography results had higher Plt/Lym ratios than patients with normal coronary angiography results. Moreover, it is also correlated with severity of coronary artery disease. Wang et $\mathrm{al}^{55}$ evaluated the Plt/Lym ratio in a study consisted of 131 patients and explored the 
association between higher Plt/Lym ratio and mortality in COVID-19 due to aggregation disorders occur in vascular structures. Relationship between Plt/Lym ratio and severity of COVID-19 was revealed in a meta-analysis included 998 patients. Higher Plt/Lym ratio on admission was found to be related with severe COVID-19. ${ }^{56}$ According to our study Plt/Lym ratio is higher in the group of patients with COVID-19 than control group.

In conclusion, the patients with COVID-19 are prone to develop cardiac morbidities and mortalities with different mechanisms. NT-proBNP, Neu/Plt, Lym/Mono and Plt/Lym ratios are the important cardiovascular markers that can be used for close follow up in hospital patients who were infected with SARS-CoV-2 and helps clinicians to adopt optimal treatment strategies at an early stage to prevent probabl cardiovascular events.

Conflicts of Interest: The authors declared no conflicts of interest.

\section{References}

1. Pranata R, Huang I, Lukito AA, Raharjo SB. Elevated N-terminal pro-brain natriuretic peptide is associated with increased mortality in patients with COVID-19: systematic review and metaanalysis.Postgrad Med $J$ 2020;96:387-391.

2. Sorrentio S, Cacia M, Leo I, Polimeni A, Sabatino J, Spaccarotella CAM, et al. B-Type Natriuretic Peptide as Biomarker of COVID-19 Disease Severity- A Meta-Analysis. J Clin Med 2020;9:1-6

3. Aladag N, Atabey RD. The role of concomitant cardiovascular diseases and cardiac biomarkers for predicting mortality in critical COVID-19 patients. ACTA Cardiologica 2020;4:1-8

4. Han H, Xie L, Liu R, Yang J, Liu F, Wu K, et al. Analysis of heart injury laboratory parameters in 273 COVID-19 patients in one hospital in Wuhan, China. J Med Virol 2020;92(7):1-5.

5. Inciardi RM, Lupi L, Zaccone G, Italia L, Raffo M, Tomasoni D, et al. Cardiac Involvement in a Patient With Coronavirus Disease 2019 (COVID-2019). JAMA Cardiology 2020;5(7):819-824.

6. Deng P, Ke Z, Ying B, Qiao B, Yuan L. The diagnostic and prognostic role of myocardial injury biomarkers in hospitalized patients with COVID-19. Clinica Chimica Acta 2020;510:186-190.

7. Shafi AA, Shaikh SA, Shirke MM, Iddawela S, Harky A. Cardiac Manifestations in COVID-19 patientsA systematic Review. J Card Surg 2020;35:1988-2008.

8. Gao L, Jiang D, Wen X, Cheng X, Sun M, He B, et al. Prognostic value of NT-pro BNP in patients with severe COVID-19. Respiratory Research 2020;21:83-89.

9. Guo T, Fan Y, Chen M, Wu X, Zhang L, He T, et al. Cardiovascular Implications of Fatal Outcomes of Patients With Coronavirus Disease 2019 (COVID-19). JAMA Card 2020;5(7):811-818.

10. Salah K, Stienen S, Pinto YM, Eurlings LW, Metra M, Genis AB, et al. Prognosis and NT-pro BNP in heart failure patients with preserved versus reduced ejection fraction. Heart 2019;105(15):1182-1189.

11. Zhang WB, Zeng YY, Wang F, Cheng L, Tang WS, Wang WQ. 2020. A high neutrophil-to-lymphocyte ratio predicts hemorrhagic transformation of large atherosclerotic infarction in patients with acute ischemic stroke. Aging 12(3):2428-2439.

12. Yayla C, Akboga MK, Yayla KG, Ertem AG, Efe TH, Sen F, et al. 2016. A novel marker of inflammation in patients with slow coronary flow: lymphocyte-to-monocyte ratio. Biomark Med 10(5):485-493.

13. Gasparyan AY, Ayvazyan L, Mukanova U, Yessirkepov M, Kitas GD. 2019. The platelet-to-lymphocyte ratio as an inflammatory marker in rheumatic diseases. Ann Lab Med 39(4):345-357.

14. Wang D, Hu B, Hu C, Zhu F, Liu X, Zhang J, et al. Clinical characteristics of 138 hospitalized patients with 2019 novel coronavirus-infected pneumonia in Wuhan, China. J Am Med Assoc2020;323:10611069.

15. Biagi A, Rossi L, Malagoli A, Zanni A, Sticozzi C, Comastri G, et al. Clinical and epidemiological characteristics of 320 deceased Covid-19 patients in an Italian provience: a retrospective observational study. J Med Virol 2020;92:2718-2724.

16. Huang C, Wang Y, Li X, Ren L, Zhao J, Hu Y. Clinical features of patients infected with 2019 novel coronavirus in Wuhan, China.Lancet 2020;395(10223):497-506.

17. Mahajan K, Negi PC, Ganju N, Asotra S. Cardiac biomarker-based risk stratification algorithm in pa- 
tients with severe COVID-19. Diabetes\&Metabolic Syndrome: Clinical Research\&ßReviews2020;14:929931.

18. Shang J, Ye G, Shi K, Wan Y, Luo C, Aihara H, et al. Structural basis receptor recognition by SARSCoV-2. Nature 2020;581:221-224.

19. Varga Z, Flammer AJ, Steiger P, Haberecker M, Andermatt R, Zinkernagel AS, et al. Endothelial cell infection and endotheliitis in COVID-19. Lancet 2020;395:1417-1418.

20. Qin JJ, Cheng X, Zhou F, Lei F, Akolkar G, Cai J, et al. Redefining Cardiac Biomarkers in Predicting Mortality of Inpatients With COVID-19. Hypertension 2020;76:1104-1112.

21. Bansal M. Cardiovascular disease and COVID-19. Diab Metab Syndrome 2020;14:247-250.

22. Fernandez AC, Izquerdo A, Subirana I, Farre N, Vila J, Duran X, et al. Markers of myocardial injury in the prediction of short-term COVID-19 prognosis. Rev Esp Cardiol 2020, article in press

23. Napoli C, Tritto I, Benincasa G, Mansueto G, Ambrosio G. Cardiovascular involvement during COVID19 and clinical implications in elderly patients. A review. Ann Med Surg 2020;57:236-243.

24. Bader F, Manla Y, Atallah B, Starling RC. Heart failure and COVID-19. Heart Failure Reviews 2021;26:1-10.

25. Kumar A, Parrillo JE, Kumar A. Clinical review: myocardial depression in sepsis and septic shock. Crit Care 2002;6(6):500-508.

26. Fung G, Luo H, Qlu Y, Yang D, McManus B. Myocarditis. Circ Res2016;118(3):496-514.

27. Massimo I, Antonio B, George L, Alessandro A, Mirko S, Allan K. Anti-inflammatory therapies for pericardial diseases in the COVID-19 pandemic: safety and potentiality. J Cardiovasc Med2020;21(9):625629.

28. Li YH, Wang MT, Huang WC, Hwang JJ. Management of acute coronary syndrome in patients with suspected or confirmed coronavirus disease 2019: Consensus from Taiwan Society of Cardiology. $J$ Formo Med Assoc 2021;120:78-82.

29. Bangalore S, Sharma A, Slotwiner A, Yatskar L, Harari R, Shah B, et al. ST-segment elevation in patients with covid-19- a case series. $N$ Engl J Med 2020;382:2478-2480.

30. Kwenander F, Japar KV, Damay V, Hariyanto TI, Tanaka M, Lugito NP, et al. Coronavirus disease 2019 and cardiovascular system: A narrative review. IJC HeartES Vasculature 2020;29:1-6.

31. Kochav SM, Coromilas E, Nalbandian A, Ranard LS, Gupta A, Chang MK, et al. Cardiac Arrhythmias in COVID-19 Infection. Circulation: Arrhythmias and Electrophysiology 2020;13(6):e008719.

32. Dherange P, Lang J, Qian P, Oberfeld B, Sauer WH, Koplan B, et al. Arrhythmias and COVID-19: A Review. JACC 2020;6(9):1193-1204.

33. Mishra AK, Sahu KK, Lal A, Sargent J. Patterns of heart injury in COVID-19 and relation to outcome. J Med Virol 2020;92:1747.

34. Weber M, Hamm C. Role of B-type natriuretic peptide (BNP) and NT-proBNP in clinical routine. Heart 2006;92(6):843-849.

35. Hall C. NT-proBNP: The Mechanism Behind the Marker. J Card Fail2005;11(5):81-83.

36. Shi S, Qin M, Shen B, Chai Y, Liu I, Yang F, et al. Association of Cardiac Injury With Mortality in Hospitalized Patients With COVID-19 in Wuhan, China. JAMA Card 2020;5:802-810.

37. Zhou F, Yu T, Du R, Fan G, Liu Y, Liu Z, et al. Clinical course and risk factors for mortality of adult inpatients with COVID-19 in Wuhan, China: a retrospective cohort study. Lancet 2020;395:1054-1062.

38. Li JW, Han TW, Woodward M, Anderson SC, Zhou H, Chan YD, et al. The impact of 2019 novel coronavirus on heart injury: a systematic review and meta-analysis. Prog Cardiovasc Dis 2020;63(4):518524.

39. He X, Wang L, Wang H, Xie Y, Yu Y, Sun J, et al. Factors associated with acute cardiac injury and their effects on mortality in patients with COVID-19. Nature 2020;10:1-9.

40. Jin L, Tang W, Song L, Luo L, Zhou Z, Fan X, et al. Acute cardiac injury in adult hospitalized COVID-19 patients in Zhuhai, China.Cardiovasc Diagn Ther 2020;10(5):1303-1312.

41. Kaplan ZS, Jackson SP. 2011. The role of platelets in atherothrombosis. Hematology Am Soc Hematol Educ Program2011(2):51-61.

42. Pearson TA, Mensah GA, Alexander RW, Anderson JL, O Cannon R, Criqui M, et al. 2003. Markers of 
inflammation and cardiovascular disease: application to clinical and public health practice: A statement for healthcare professionals from the Centers for Disease Control and Prevention and the American Heart Association. Circulation 107(3):499-511.

43. Gong S, Gao X, Xu F, Shang Z, Li S, Chen W, et al. 2018. Association of lymphocyte-to-monocyte ratio with severity of coronary artery disease. Med (Baltimore) 97(43):e12813.

44. Forget P, Kahfia C, Defour JP, Latinne D, VanPel MC, De Kock M. 2017. What is the normal value of the neutrophil-to-lymphocyte ratio? BMC Research Notes 10(12):233-238.

45. Balta S, Celik T, Mikhailidis DP, Ozturk C, Demirkol S, Aparci M, et al. 2016. The relation between atherosclerosis and the neutrophil-lymphocyte ratio. Clin Appl ThrombHemost22(5):405-411.

46. Kaya H, Ertas H, Islamoglu Y, Kaya Z, Atilgan ZA, Cil H, et al. 2014. Association between neutrophil to lymphcyte ratio and severity of coronary artery disease. Clin Appl ThrombHemost 20(1):50-54.

47. Liu Y, Du X, Chen J, Jin Y, Peng L, Wang HH, et al. Neutrophil-to-lymphocyte ratio as an independent risk factor for mortality in hospitalized patients with COVID-19. Journal of Infection 2020;81(1):6-12.

48. Tatum D, Taghavi S, Houghton A, Stover J, Torah E, Duchesne J. Neutrophil-to-Lymphocyte Ratio and Outcomes in Louisiena Covid-19 Patients. Shock 2020;54(5):652-658.

49. Gary T, Pichler M, Belaj K, Eller P, Hafner F, Gerger A, et al. Lymphocyte-to-monocyte ratio: a novel marker for critical limb ischemia in PAOD patients. Int $J$ Clin Pract 2014;68(3):1483-1487.

50. Demir V, Akboga MK, Yilmaz S, Ede H. Assessment of monocyte-to-high density lipoprotein cholesterol ratio and lymphocyte-to-monocyte ratio in patients with metabolic syndrome. Biomark Med2017;11(7):535-540.

51. Lissoni P, Rovelli F, Monzon A, Privitera C, Messina G, Porro G, et al. Evidence of Abnormally Low Lymphocyte-To-Monocyte Ratio In Covid-19-Induced Severe Acute Respiratory Syndrome. J Immuno Allerg 2020;1(2):1-6.

52. Shivakumar BG, Gosavi S, Rao AA, Shastry S, Raj SS, Sharma A, et al. Neutrophil-to-Lymphocyte, Lymphocyte-to-Monocyte and Platelet-to-Lymphocyte Ratios: Prognostic Significance in COVID19. Cureus 2021;13(1):e12622.

53. Kurtul A, Sani NM, Yarlioglues M, Duran M, Ergun G, Acikgoz SK, et al. Association of plateletto-lymphocyte ratio with severity and complexity of coronary artery disease in patients with acute coronary syndrome. Am J Cardiol 2014;114(7):972-978.

54. Sari I, Sunbul M, Mammadov C, Durmus E, Bozbay M, Kivrak T, et al. Relation of neutrophil-tolymphocyte and platelet-to-lymphocyte ratio with coronary artery disease severity in patients undergoing coronary angiography. Kardiol Pol 2015;73(12):1310-1316.

55. Wang X, Li X, Shang Y, Wang J, Zhang X, Su D, et al. Ratios of neutrophil-to-lymphocyte and plateletto-lymphocyte predict all-cause mortality in inpatients with coronavirus disease 2019 (COVID-19): a cohort study in a single medical center. Epidemiology and Infection 2020;148:211-217.

56. Simadibrata DM, Pandhita BA, Ananta ME, Tango T. Platelet-to-lymphocyte ratio, a novel biomarker to predict the severity of COVID-19 patients: A systemic review and meta-analysis. $J$ Int $C$ Soc 2020;0(0):1-7.

Table 1. Demographic features and laboratory findings of the groups

\begin{tabular}{llll}
\hline Parameters & Controls $(\mathrm{n}=33)$ & $\begin{array}{l}\text { Patients with COVID-19 } \\
(\mathrm{n}=33)\end{array}$ & P values \\
\hline Age $^{\mathrm{a}}$ & $58.33 \pm 1.70$ & $58.48 \pm 3.01$ & 0.9655 \\
Gender $(\mathrm{n}, \%)$ Male & $18(54.55) 15(45.45$ & $20(60.60) 13(39.40)$ & 0.8033 \\
Female & & & \\
NT-proBNP $(\mathrm{pg} / \mathrm{ml})$ & $149.32 \pm 16.33$ & $1262.67 \pm 394.93$ & $<\mathbf{0 . 0 0 0 1}$ \\
WBC $\left(10^{3} / \mathrm{uL}\right)$ & $7.75 \pm 0.28$ & $6.98 \pm 0.44$ & 0.1467 \\
Neutrophils $\left(10^{3} / \mathrm{uL}\right)$ & $4.48 \pm 0.23$ & $5.14 \pm 0.42$ & 0.1782 \\
Lymphocytes $\left(10^{3} / \mathrm{uL}\right)$ & $2.40 \pm 0.11$ & $1.29 \pm 0.09$ & $<\mathbf{0 . 0 0 0 1}$ \\
Monocytes $\left(10^{3} / \mathrm{uL}\right)$ & $0.63 \pm 0.03$ & $0.50 \pm 0.04$ & $\mathbf{0 . 0 4 3 7}$ \\
Platelets $\left(10^{3} / \mathrm{uL}\right)$ & $265.67 \pm 11.45$ & $525.76 \pm 36.96$ & 0.2852
\end{tabular}




\begin{tabular}{llll}
\hline Parameters & Controls $(\mathrm{n}=33)$ & $\begin{array}{l}\text { Patients with COVID-19 } \\
(\mathrm{n}=33)\end{array}$ & P values \\
\hline AST (U/L) & $28.09 \pm 1.53$ & $42.27 \pm 7.55$ & 0.4302 \\
ALT (U/L) & $26.79 \pm 1.01$ & $45.61 \pm 9.52$ & 0.4378 \\
AST /ALT & $1.04 \pm 0.03$ & $1.11 \pm 0.07$ & 0.3349 \\
Creatinine (mg/dl) & $0.72 \pm 0.03$ & $0.82 \pm 0.04$ & 0.0543 \\
D-Dimer (ng/ml) & $0.54 \pm 0.03$ & $0.83 \pm 0.11$ & $\mathbf{0 . 0 1 3 5}$ \\
CRP (mg/L) & $0.55 \pm 0.03$ & $54.90 \pm 11.25$ & $<\mathbf{0 . 0 0 0 1}$ \\
Neutrophil-to- & $2.04 \pm 0.18$ & $4.55 \pm 0.49$ & $<\mathbf{0 . 0 0 0 1}$ \\
Lymphocyte & & & $<\mathbf{0 . 0 0 0 1}$ \\
Lymphocyte-to- & $4.17 \pm 0.27$ & $2.71 \pm 0.20$ & $<\mathbf{0 . 0 0 0 1}$ \\
Monocyte & & & \\
Platelet-to- & $120.24 \pm 9.11$ & $215.04 \pm 20.60$ & \\
Lymphocyte & & & \\
\hline
\end{tabular}

${ }^{\mathrm{a}}$ Data are presented as mean $\pm \mathrm{SEM}$.

NT-pro BNP: N-Terminal pro-Brain Natriuretic Peptide, WBC: White Blood Cell,

AST: Aspartate Aminotransferase, ALT: Alanine Aminotransferase

CRP: C-reactive protein

Table 2. Correlations between biochemicalparametersin COVID-19 patients.

\begin{tabular}{llll}
\hline Parameters & $\begin{array}{l}\text { Correlation coefficient } \\
(\mathbf{r})\end{array}$ & $\begin{array}{l}\text { Coefficient of } \\
\text { determination }\left(\mathbf{r}^{2}\right)\end{array}$ & $\mathbf{P}$ \\
\hline $\begin{array}{l}\text { NT-proBNP- } \\
\text { Lymphocytes }\end{array}$ & -0.0498 & 0.0025 & 0.7831 \\
NT-proBNP- & 0.0510 & 0.0026 & 0.7781 \\
$\begin{array}{l}\text { Monocytes } \\
\text { NT-proBNP-D-Dimer }\end{array}$ & 0.6780 & 0.4597 & $<\mathbf{0 . 0 0 0 1}$ \\
NT-proBNP-CRP & 0.1722 & 0.0297 & 0.3378 \\
NT-proBNP- & 0.1608 & 0.0259 & 0.3714 \\
$\begin{array}{l}\text { Neutrophil-to- } \\
\text { Lymphocyte }\end{array}$ & & \\
$\begin{array}{l}\text { NT-proBNP- } \\
\text { Lymphocyte-to- }\end{array}$ & -0.1272 & 0.0162 & 0.4806 \\
$\begin{array}{l}\text { Monocyte } \\
\text { NT-proBNP-Platelet- }\end{array}$ & 0.1103 & & \\
to-Lymphocyte & & 0.0122 & 0.5412 \\
\hline
\end{tabular}

NT-pro BNP: N-Terminal pro-Brain Natriuretic Peptide, CRP: C Reactive Protein

Figure Legends

Fig 1. Comparison of NT-proBNP levels between patients with COVID-19 and controls

NT-pro BNP: N-Terminal pro-Brain Natriuretic Peptide

\section{Hosted file}

Fig IJCP.pdf available at https://authorea.com/users/405703/articles/516586-comparison-of- 
the-n-terminal-pro-brain-natriuretic-peptide-levels-neutrophil-to-lymphocyte-lymphocyteto-monocyte-and-platelet-to-lymphocyte-ratios-between-the-patients-with-covid-19-andhealthy-subjects-are-the-patients-with-covid-19-under-the-risk-of-cardiovascular-events 